\title{
Eating with a smaller spoon decreases bite size, eating rate and ad libitum food intake in healthy young males
}

\author{
Lewis J. James ${ }^{1 *}$, Tyler Maher ${ }^{2,3}$, Jack Biddle ${ }^{2}$ and David R. Broom ${ }^{2}$ \\ ${ }^{1}$ National Centre for Sport and Exercise Medicine, School of Sport, Exercise and Health Sciences, Loughborough University, \\ Loughborough LE11 3TU, UK \\ ${ }^{2}$ Academy of Sport and Physical Activity, Sheffield Hallam University, Sheffield S1O 2BP, UK \\ ${ }^{3}$ Department of Sport, Health Sciences and Social Work, Faculty of Health and Life Sciences, Oxford Brookes Centre \\ for Nutrition and Health, Oxford Brookes University, Gipsy Lane, Oxford OX3 OBP, UK
}

(Submitted 5 January 2018 - Final revision received 13 June 2018 - Accepted 19 July 2018 - First published online 29 August 2018)

\section{Abstract}

There is a paucity of data examining the effect of cutlery size on the microstructure of within-meal eating behaviour or food intake. Therefore, the present studies examined how manipulation of spoon size influenced these eating behaviour measures in lean young men. In study one, subjects ate a semi-solid porridge breakfast ad libitum, until satiation. In study two, subjects ate a standardised amount of porridge, with mean bite size and mean eating rate covertly measured by observation through a one-way mirror. Both studies involved subjects completing a familiarisation visit and two experimental visits, where they ate with a teaspoon (SMALL) or dessert spoon (LARGE), in randomised order. Subjective appetite measures (hunger, fullness, desire to eat and satisfaction) were made before and after meals. In study one, subjects ate $8 \%$ less food when they ate with the SMALL spoon (SMALL 532 (sD 189) g; LARGE 575 (sD 227) g; $P=0 \cdot 006$ ). In study two, mean bite size (SMALL 10.5 (sD 1.3) g; LARGE 13.7 (sD 2.6) g; $P<0.001$ ) and eating rate (SMALL 92 (sD 25) g/min; LARGE 108 (sD 29) g/min; $P<0.001$ ) were reduced in the SMALL condition. There were no condition or interaction effects for subjective appetite measures. These results suggest that eating with a small spoon decreases ad libitum food intake, possibly via a cascade of effects on within-meal eating microstructure. A small spoon might be a practical strategy for decreasing bite size and eating rate, likely increasing oral processing, and subsequently decreasing food intake, at least in lean young men.

\section{Key words: Appetite: Energy balance: Weight gain: Obesity: Portion size}

Obesity, the result of chronic positive energy balance, continues to rise ${ }^{(1,2)}$, representing a major health and economic burden on society. Increases in portion size are believed to contribute to excess energy intake (i.e. energy intake greater than energy expenditure), and recent evidence suggests that reducing portion size can decrease food intake ${ }^{(3)}$. Manipulation of the eating environment, and specifically tableware, is one strategy that has been used to reduce portion size ${ }^{(3,4)}$. Whilst the impact of dishware size (i.e. plate or bowl size) on food intake has been well studied, albeit with varied findings ${ }^{(4)}$, the role of cutlery size has received little attention.

Manipulating the microstructure of within-meal eating behaviour (e.g. bite size, eating rate etc.) might independently or interactively influence food intake. A recent meta-analysis of intervention studies reported that a faster eating rate was associated with increased ad libitum food intake compared with eating more slowly ${ }^{(5)}$. Similarly, bite or sip size has been shown to influence food intake, with smaller bites or sips decreasing ad libitum intake ${ }^{(6-8)}$, possibly via an interaction with eating rate ${ }^{(9,10)}$. Intuitively, manipulation of cutlery size might influence bite size by altering the amount of food presented to the mouth, potentially influencing eating rate and food intake. Indeed, Geier et al. ${ }^{(11)}$ reported that increasing the size of a spoon used to serve chocolate confectionary increased the amount of food served, but little is known about how cutlery used to eat meals influences food intake.

Previous studies have used smaller cutlery (namely spoons) as part of a combined strategy (including instructions to eat slowly, chew the food more, put the spoon down between bites etc.) to reduce eating rate ${ }^{(12,13)}$. However, the combination of strategies used makes it difficult to discern the specific effects of cutlery size on eating behaviour. Mishra et $a l .^{(14)}$ is, to our knowledge, the only study to directly examine the effect of cutlery size on ad libitum food intake. Mishra et al. ${ }^{(14)}$ reported that in a controlled laboratory environment, eating with a small fork decreased ad libitum food intake, but the reverse was

Abbreviations: DTE, desire to eat; LARGE, dessert spoon; SMALL, teaspoon.

* Corresponding author: L. J. James, email L.James@lboro.ac.uk 
reported (i.e. a smaller fork increased food intake) when meals were consumed in a habitual restaurant setting. The authors attribute this disparity between settings to goal-effort links pertaining to the eating environment, although interpretation of the results from the restaurant is complicated by the uncontrolled conditions present (i.e. the different meals selected, starters eaten, variety of drinks available/consumed, dessert planned, social interactions etc.), making firm conclusions difficult to make. How manipulation of cutlery size influences the microstructure of within-meal eating behaviour is currently unknown.

Due to the paucity and inconsistency of data examining the influence of cutlery size on within-meal eating behaviour, the present studies aimed to compare the effects of eating a semisolid breakfast with a teaspoon (small spoon) or dessert spoon (large spoon) on (1) ad libitum food intake (study one) and (2) the microstructure of within-meal eating behaviour including bite size, eating rate and meal duration (study two). It was hypothesised that eating with a small spoon would reduce ad libitum food intake in study one and that eating with a small spoon would reduce bite size and eating rate, as well as increasing meal duration in study two.

\section{Methods}

\section{Overview of experimental protocol}

This investigation comprised two separate studies, which were conducted according to the guidelines laid down in the Declaration of Helsinki and all procedures were approved by the Loughborough University Ethics Approvals (Human Participants) Sub-Committee and Sheffield Hallam University Faculty of Health and Wellbeing Ethics Committee (R13-P7; C15-34). Data for study one were collected at both institutions, whilst data for study two were only collected at Loughborough University. Written informed consent was obtained from all subjects before participation. During both studies, subjects completed a familiarisation trial, followed by two experimental trials completed in a randomised order and separated by $\geq 7 \mathrm{~d}$. Randomisation was undertaken before the start of data collection. During experimental trials, subjects consumed an ad libitum (study one) or a standardised (study two) breakfast meal with a teaspoon (SMALL) or dessert spoon (LARGE). Spoons were from the same cutlery set (Tesco Value; Tesco) and thus, except for size, were identical in appearance. The SMALL and LARGE spoons were 146 and $194 \mathrm{~mm}$ in length, respectively, and had heads that were roughly oval in shape. The length and width of the SMALL spoon's head were 46 and $31 \mathrm{~mm}$, respectively, whilst the length and width of the LARGE spoon's head were 61 and $42 \mathrm{~mm}$, respectively. The estimated surface area of the SMALL spoon's head was approximately 39\% less than the LARGE spoon's head (i.e. approximately $1230 \mathrm{~mm}^{2} v$. approximately $2030 \mathrm{~mm}^{2}$ ).

\section{Subjects}

In all, twenty-nine men (age 24 (SD 4) years, height 1.77 (sD 0.06 ) $\mathrm{m}$, body mass 73.7 (sD 8.8) kg, BMI 23.5 (sD 2.4) kg/m², body fat 17 (SD 4)\%) completed study one, whilst sixteen men (age 27 (sD 3) years, height 1.82 (sD 0.06) m, body mass 79.9 (SD 9.9) $\mathrm{kg}$, BMI 24.0 (SD 1.9) $\mathrm{kg} / \mathrm{m}^{2}$, body fat 15 (SD 3)\%) completed study two. For inclusion, subjects were required to be male, with a BMI $<30 \mathrm{~kg} / \mathrm{m}^{2}$ and body fat $<25 \%$, be generally fit and healthy with no acute or chronic morbidity known to influence appetite/food intake and had to not score in the clinical range for dietary restraint, disinhibition or hunger, as measured by the Three Factor Eating Questionnaire ${ }^{(15)}$. In all, eight subjects had a BMI $>25 \mathrm{~kg} / \mathrm{m}^{2}$ (range $\left.25 \cdot 2-27 \cdot 9 \mathrm{~kg} / \mathrm{m}^{2}\right)$ in study one, whilst four subjects had a BMI $>25 \mathrm{~kg} / \mathrm{m}^{2}$ (range $25 \cdot 4-27 \cdot 3 \mathrm{~kg} / \mathrm{m}^{2}$ ) in study two.

\section{Pretrial standardisation}

In both studies, subjects recorded all food and drink consumed, as well as any low intensity habitual physical activity undertaken in the $24 \mathrm{~h}$ before the first experimental trial. They were then asked to replicate these diet and activity patterns in the $24 \mathrm{~h}$ before the second experimental trial. Subjects were asked to refrain from moderate or strenuous physical activity and alcohol intake during this $24 \mathrm{~h}$ period. All trials commenced in the morning after an overnight fast (07.00-10.00 hours), with the time standardised for each subject.

\section{Familiarisation trials}

During both studies, subjects initially completed a familiarisation trial before experimental trials. At this visit, subjects' height and body mass were determined, before subcutaneous skinfold measurements were obtained from the triceps, biceps, subscapular and suprailiac for estimation of body fat percentage ${ }^{(16)}$. Subjects were then familiarised with the methods used in experimental trials, by undertaking a practice trial identical in procedure to the experimental trials, during which the LARGE spoon was used to eat.

\section{The breakfast meal}

Porridge was used as a breakfast meal in both studies. In study one, three flavours of porridge were available (plain, chocolate and golden syrup), with subjects choosing their preferred flavour before the familiarisation trial and eating this flavour during all subsequent trials. The meal was made by mixing a commercially available porridge oat mix (Ready Brek; Weetabix) with semiskimmed milk (Tesco) in a ratio of $90 \mathrm{~g}$ oats: $420 \mathrm{ml}$ milk. In study two, all subjects were provided with the plain porridge, sweetened with sugar, with a ratio of $72 \mathrm{~g}$ oats: $18 \mathrm{~g}$ sugar: $420 \mathrm{ml}$ milk. In each study, all meals were prepared using standardised operating procedures to ensure identical temperature, texture and flavour for each subject for each trial, with bowls and spoons weighed before preparation, as well as before and after eating to determine food consumption.

\section{Study one experimental protocol}

Subjects consumed an ad libitum porridge breakfast in each trial and were given standardised instructions to 'eat until you are comfortably full and satisfied'. The meal was served in a 
custom-made eating booth to minimise external distractions and to allow experimenters to supply food to subjects with minimal interaction. Subjects were provided with a bowl of porridge and a spoon to eat with. They ate until they had consumed approximately half to three-quarters of the bowl (time taken to do this was determined during the familiarisation trial), when another bowl was supplied. This pattern continued until subjects were satiated. The eating booth was situated inside a larger eating laboratory (still devoid of food cues) and subjects left the booth but remained inside the laboratory once satiated. They remained inside the laboratory for the duration of the 30-min eating period and could return to the eating booth and continue eating if they desired. Each subject was in isolation in the eating laboratory during each 30-min eating period, with only essential interaction between experimenter and subject for the delivery of food and water at predetermined time points. Water was available ad libitum throughout the meal, with glasses weighed before and after the meal to determine the amount consumed. Before and after the 30-min eating period, subjects provided ratings of hunger, fullness, desire to eat (DTE) and satisfaction.

To blind subjects to the true aim of the study they were told the purpose of the study was to assess the reproducibility of the ad libitum breakfast meal. This information was disseminated to subjects through a written information sheet that they read before consenting to take part in the study. This was reaffirmed by an experimenter verbally explaining the study design and the purpose (i.e. to examine reproducibility of the meal). At the end of the study, subjects were asked three exit questions: 'Did you think the meals were similar in texture/taste', 'Do you think the eating environment was similar between trials?' and 'Do you have any other comments?'. These questions gave subjects the opportunity to indicate if they had noticed the difference in spoon size between trials

\section{Study two experimental protocol}

Subjects were provided with a standardised porridge meal providing $15 \%$ of estimated daily energy requirements, which were determined using their predicted resting metabolic rate ${ }^{(17)}$ multiplied by a physical activity level of $1 \cdot 5$. The meal was consumed in an observation laboratory, which included a section of one-way mirror, so an experimenter could observe the subject whilst they ate. The meal was served in a single bowl and subjects were instructed to 'eat until you have finished the bowl'. During eating, the same experimenter recorded each time the subject took a spoonful of porridge from the bowl and each time they took a mouthful (bite) of porridge from the spoon. The total time taken to eat the meal was also recorded. Before and immediately after finishing the meal, subjects provided ratings of hunger, fullness, DTE and satisfaction, with a final rating taken for $15 \mathrm{~min}$ after starting the meal. No water was consumed during the meal. Again, each subject was in isolation in the eating laboratory during each 15-min eating period, with only essential interaction between experimenter and subject for the delivery of food and appetite questionnaires.

Mean eating rate $(\mathrm{g} / \mathrm{min})$ was determined by dividing the total weight of porridge consumed by the time taken to eat the meal. Mean bite size (g) was determined by dividing the total weight of porridge consumed by the number of bites taken to eat the porridge.

To blind subjects to the true aim of the study they were told the purpose of the study was to assess the subjective appetite response to eating with different size spoons. Subjects were not aware they were being observed.

\section{Subjective appetite sensations}

Subjects completed visual analogue scale questionnaires to assess their hunger 'How hungry do you feel now?', fullness 'How full do you feel now?', DTE 'how much would you like to eat a meal now?' and satisfaction 'How satisfied do you feel now?'. Questions were administered on a $100 \mathrm{~mm}$ lines, with the verbal anchors 'not at all' and 'very' at 0 and $100 \mathrm{~mm}$, respectively. These questionnaires have previously been shown to be reliable for use in appetite research ${ }^{(18)}$.

\section{Sample size}

An $\alpha$ of 0.05 and a $\beta$ of 0.2 were used to estimate the required sample size for each study. For study one, previous data from our laboratory $^{(19)}$ were used to estimate food intake and a between group correlation of 0.9 estimated that twenty-five subjects would be required to detect an $8 \%$ difference in food intake, providing an estimated effect size $\left(d_{z}\right)$ of 0.59 . For study two, approximate eating rates and the between group correlation of 0.94 observed in study one were used to estimate sixteen subjects would be required to detect an $8 \%$ difference in mean eating rate, providing an estimated effect size $\left(d_{z}\right)$ of 0.77 .

\section{Statistical analysis}

All data were analysed using IBM SPSS Statistics 23. All data were initially checked for normality of distribution using a Shapiro-Wilk test. Subjective appetite data were analysed using two-way repeated-measures ANOVA. Where the assumption of sphericity was violated the df were corrected using the Greenhouse-Geisser estimate. Data containing one factor were analysed using paired $t$ tests (normally distributed data) or Wilcoxon signed-rank tests (non-normally distributed data). Effect sizes (Cohen's $d_{z}$ ) were calculated for paired comparisons. Relationships between variables were explored using Pearson's product-moment correlation coefficient or Spearman's rank correlation coefficient, as appropriate. Differences were accepted as being significant when $P \leq 0.05$ and all data are presented as means and standard deviations unless otherwise stated.

\section{Results}

Study one

Ad libitum food intake. The amount of food consumed during the ad libitum meal was $8 \%$ less when subjects ate with the small spoon compared with the large spoon (SMALL 532 (SD 189) g, LARGE 575 (sD 227) g; $Z=-2.692 ; d_{z}=0.55 ; P=0.006$; Fig. 1(a)), whilst water drunk with the meal was similar between 

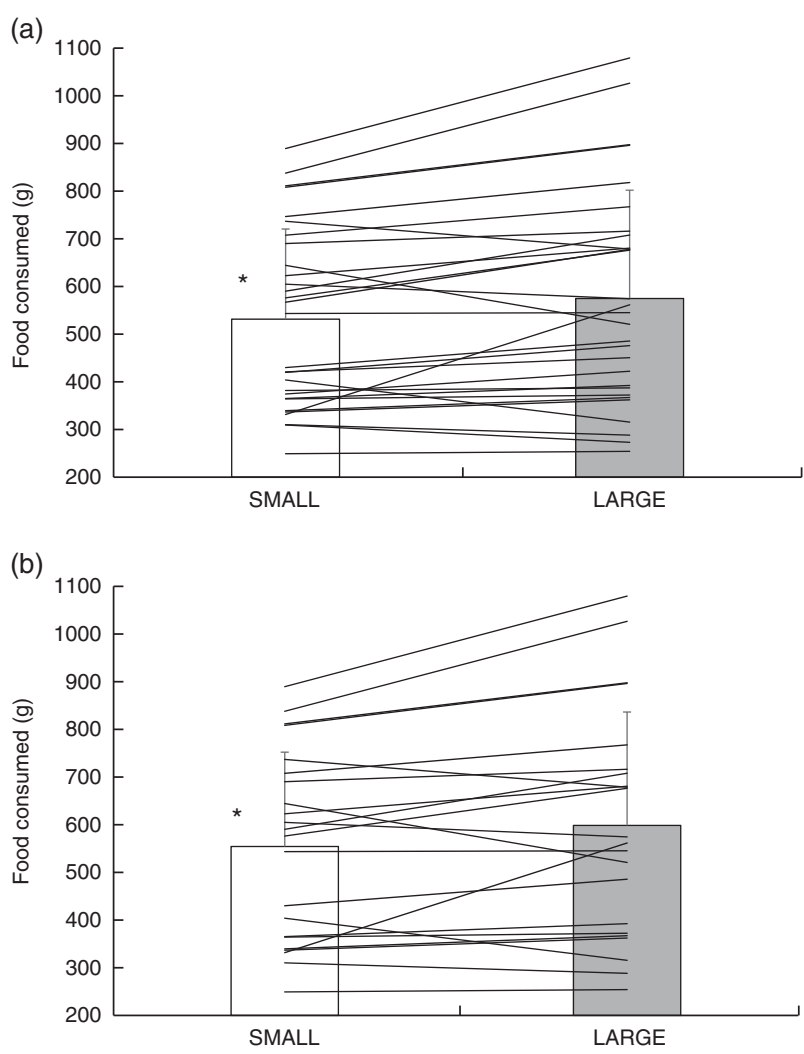

Fig. 1. (a) Ad libitum food consumed during study one for all subjects and (b) for subjects who did not report an awareness of the difference in spoon size between conditions. Values are means, with standard deviations represented by vertical bars. Lines are individual subject data. * Significantly different between trials. SMALL, teaspoon; LARGE, dessert spoon.

trials (SMALL (362 (SD 130) g; LARGE 325 (SD 129) g; $t=1.454$; $\left.d_{z}=-0 \cdot 27 ; P=0 \cdot 157\right)$.

Subjective appetite. Due to an issue with one appetite questionnaire on one trial for one subject, the results for twentyeight subjects are presented. There were main effects of time for hunger $\quad\left(F_{1,27}=574.336 ; \quad P<0.001 ; \quad\right.$ Table 1$)$, fullness $\left(F_{1,27}=640.587 ; \quad P<0.001 ;\right.$ Table 1$)$, DTE $\left(F_{1,27}=688.796\right.$; $P<0.001$; Table 1$)$ and satisfaction $\left(F_{1,27}=312 \cdot 917 ; P<0 \cdot 001\right.$; Table 1), with hunger and DTE decreasing, and fullness and satisfaction increasing over the meal. However, there were no main effects of trial (hunger $F_{1,27}=0.547 ; P=0.466$; fullness $F_{1,27}=0 \cdot 159 ; P=0.693 ;$ DTE $F_{1,27}=0.939 ; P=0.341 ;$ satisfaction $F_{1,15}=1 \cdot 191 ; \quad P=0.285$ ), or interaction effects (hunger $F_{1,27}=0.005 ; P=0.945 ;$ fullness $F_{1,27}=0.473 ; P=0.497 ;$ DTE $F_{1,27}=0 \cdot 149 ; P=0 \cdot 703 ;$ satisfaction $\left.F_{1,27}=0.989 ; P=0.329\right)$.

Study blinding. In all, seven subjects (24\%) identified that the spoons used in the two experimental trials were different sizes during the exit questions. When the seven subjects who reported an awareness of the difference in spoon size between conditions were removed, ad libitum food consumption was still approximately $8 \%$ less in the small spoon condition (SMALL 554 (sD 198) g, LARGE 599 (sD 238) g; $t=-2.364 ; d_{z}=0.54$; $P=0 \cdot 028$; Fig. 1(b))

\section{Study two}

Eating behaviour. The amount of residual porridge remaining on the bowl and spoon at the end of the meal was similar between trials $\left(Z=-0.085 ; d_{z}=0.14 ; P=0.932\right.$; Table 2), and consequently, the amount of porridge consumed was also similar between trials $\left(t=0.122 ; d_{z}=0.03 ; P=0.904\right.$; Table 2$)$.

The number of spoonfuls $\left(Z=-3.520 ; d_{z}=2.03 ; P<0.001\right.$; Table 2) and bites $\left(Z=-3.519 ; d_{z}=2.00 ; P<0.001\right.$; Table 2), as well as the total time $\left(t=4 \cdot 078 ; d_{z}=-1.05 ; P<0 \cdot 001\right.$; Table 2) taken to eat the meal were all greater during the SMALL trial. In both trials, there was a strong correlation between the number of spoonfuls and bites used to eat the meal (SMALL $r$ 0.991; $P<0.001$; LARGE $r 0.968 ; P<0 \cdot 001$ ), with eleven out of sixteen subjects using an identical number of spoonfuls and bites in both trials. Consequently, mean bite size $\left(t=-6 \cdot 155 ; d_{z}=1.59\right.$; $P<0.001$; Fig. 2(a) $)$ and eating rate $\left(Z=-3.258 ; d_{z}=1 \cdot 04\right.$; $P=0.001$; Fig. 2(b)) were lower during the SMALL trial. There were positive correlations between the change in bite size and change in eating rate, when represented as absolute $(r 0.612$; $P=0.012$; Fig. 3) or relative $(r 0.613 ; P=0.012)$ values.

Subjective appetite. There were main effects of time for hunger $\left(F_{1.094,16.403}=66.761 ; \quad P<0.001 ;\right.$ Table 1$)$, fullness $\left(F_{1 \cdot 193,17.902}=116.390 ; \quad P<0.001 ; \quad\right.$ Table 1$), \quad$ DTE $\left(F_{1.068,16.021}=98.587 ; \quad P<0.001 ; \quad\right.$ Table 1$)$ and satisfaction $\left(F_{1 \cdot 116,16.737}=106.283 ; \quad P<0.001 ;\right.$ Table 1$)$, with hunger and DTE decreasing and fullness and satisfaction increasing over the meal. However, there were no main effects of trial (hunger $F_{1,15}=0.010 ; P=0.923 ;$ fullness $F_{1,15}=3.587 ; P=0.078 ;$ DTE $F_{1,15}=0.037 ; P=0.851 ;$ satisfaction $F_{1,15}=2 \cdot 402 ; P=0 \cdot 142$ ), or interaction effects (hunger $F_{2,30}=0.911 ; P=0.413$; fullness $F_{2,30}=0.661 ; P=0.524 ;$ DTE $F_{2,30}=0.461 ; P=0.635$; satisfaction $\left.F_{2,30}=1.437 ; P=0.253\right)$.

\section{Discussion}

These studies aimed to examine the effect of manipulating cutlery size (i.e. spoon size) on ad libitum food intake (study one) and the microstructure of within-meal eating behaviour (specifically bite size, eating rate and meal duration; study two) using a semi-solid breakfast food (porridge) in lean young men. The main finding from study one was that eating with the small spoon resulted in a small but statistically significant (approximately $8 \%$ ) decrease in ad libitum food intake. The main findings from study two were that subjects used more spoonfuls, used more bites and took more time to finish the standardised meal when they ate with the small spoon. These findings meant that both mean bite size and mean eating rate were less when subjects ate with the small spoon.

Prolonged positive energy balance (i.e. energy intake greater than energy expenditure) results in accumulation of energy within the body, principally in adipose tissue, and ultimately leads to obesity. As the prevalence of obesity continues to rise both in the $\mathrm{UK}^{(1)}$ and around the globe ${ }^{(2)}$, strategies that reduce energy balance become increasingly important. Clearly, reducing energy intake by moderating portion size is one such 
Table 1. Hunger $(\mathrm{mm})$, fullness $(\mathrm{mm})$ and satisfaction $(\mathrm{mm})$ before and after the fixed meal periods for both study one ( $30 \mathrm{~min})$ and study two (15 min), as well as immediately after finishing eating the standardised meal in study two

(Mean values and standard deviations)

\begin{tabular}{|c|c|c|c|c|c|c|}
\hline & \multicolumn{2}{|c|}{$\begin{array}{c}\text { Before } \\
\text { meal period }\end{array}$} & \multicolumn{2}{|c|}{$\begin{array}{l}\text { Immediately after } \\
\text { eating }\end{array}$} & \multicolumn{2}{|c|}{ After meal period } \\
\hline & Mean & SD & Mean & SD & Mean & SD \\
\hline \multicolumn{7}{|c|}{ Study one: hunger (mm) } \\
\hline SMALL & 76 & 15 & - & - & 5 & 5 \\
\hline LARGE & 75 & 15 & - & - & 4 & 5 \\
\hline \multicolumn{7}{|c|}{ Study one: fullness (mm) } \\
\hline SMALL & 17 & 13 & - & - & 89 & 8 \\
\hline LARGE & 16 & 11 & - & - & 89 & 8 \\
\hline \multicolumn{7}{|c|}{ Study one: desire to eat $(\mathrm{mm})$} \\
\hline SMALL & 82 & 11 & - & - & 7 & 11 \\
\hline LARGE & 81 & 13 & - & - & 5 & 6 \\
\hline \multicolumn{7}{|c|}{ Study one: satisfaction (mm) } \\
\hline SMALL & 22 & 13 & - & - & 86 & 16 \\
\hline LARGE & 22 & 17 & - & - & 90 & 8 \\
\hline \multicolumn{7}{|c|}{ Study two: hunger (mm) } \\
\hline SMALL & 74 & 19 & 20 & 15 & 16 & 15 \\
\hline LARGE & 75 & 21 & 18 & 14 & 17 & 15 \\
\hline \multicolumn{7}{|c|}{ Study two: fullness (mm) } \\
\hline SMALL & 19 & 15 & 78 & 9 & 78 & 14 \\
\hline LARGE & 15 & 14 & 74 & 14 & 77 & 15 \\
\hline \multicolumn{7}{|c|}{ Study two: desire to eat (mm) } \\
\hline SMALL & 82 & 18 & 19 & 17 & 17 & 16 \\
\hline LARGE & 78 & 17 & 20 & 13 & 19 & 15 \\
\hline \multicolumn{7}{|c|}{ Study two: satisfaction (mm) } \\
\hline SMALL & 25 & 17 & 81 & 16 & 82 & 14 \\
\hline LARGE & 19 & 17 & 80 & 16 & 80 & 16 \\
\hline
\end{tabular}

SMALL, teaspoon; LARGE, dessert spoon.

Table 2. Food consumption and within-meal eating behaviour variables for study two

(Mean values and standard deviations)

\begin{tabular}{lccccc}
\hline & \multicolumn{2}{c}{ SMALL } & & \multicolumn{2}{c}{ LARGE } \\
\cline { 2 - 3 } \cline { 6 - 7 } & Mean & SD & & Mean & SD \\
\hline Food eaten (g) & $375 \cdot 5$ & 27.4 & & $375 \cdot 6$ & $29 \cdot 1$ \\
Food left (g) & 2.6 & 1.9 & & 2.5 & 1.2 \\
Spoons used & 36 & 5 & & $28^{*}$ & 6 \\
Bites used & 36 & 5 & & $28^{*}$ & 6 \\
Meal duration (min) & $4 \cdot 3$ & 0.8 & & $3 \cdot 7^{*}$ & 0.8
\end{tabular}

SMALL, teaspoon; LARGE, dessert spoon

* Significantly different between trials.

strategy that might assist with energy balance control. The results for ad libitum food intake (i.e. study one) are consistent with those of a similar controlled laboratory experiment, reporting that eating with a smaller fork reduced food intake from an ad libitum pasta meal ${ }^{(14)}$. Interestingly, Mishra et al. ${ }^{(14)}$ also reported the reverse response in an uncontrolled restaurant setting (i.e. those who ate with the larger fork ate less). The authors suggest the disparity in findings between laboratory and restaurant settings relate to the presence of a well-defined goaleffort link in the restaurant setting. However, the lack of control between groups (i.e. small/large fork) in the restaurant study for the meal selected, starters eaten, variety of drinks available/ consumed, dessert planned, social interactions, etc., make the findings difficult to interpret. It seems, when tested in a controlled laboratory environment, that reducing cutlery size decreases food intake, but further work is needed to explore other eating occasions and environments to better understand the effects. Nonetheless, the finding that ad libitum food intake is reduced when the food is eaten with a smaller spoon is intriguing as it suggests using smaller cutlery might offer a simple practical strategy to help moderate daily energy intake.

In study two, we investigated some of the potential mechanisms by which manipulating cutlery size might influence ad libitum food intake. Accumulating evidence suggests that oral processing might represent an important factor governing food intake, with increased oral processing (i.e. increased orosensory exposure), increasing satiation ${ }^{(9)}$. Two inter-related elements of within-meal eating microstructure that might influence oral processing are bite size and eating rate.

The results of study two demonstrate that eating with a small spoon increases the number of spoonfuls used to eat the meal, consequently reducing bite size by approximately $24 \%$. Although not a universal finding ${ }^{(20)}$, reducing bite/sip size of a food/liquid has been shown to decrease ad libitum intake ${ }^{(6-8)}$. For example, Zijlstra et al. ${ }^{(7)}$ reported an approximately $18 \%$ decrease in ad libitum intake of a chocolate custard when bite size was reduced from 15 to $5 \mathrm{~g}$ (i.e. approximately $67 \%$ reduction). Similarly, reducing sip size of soup by approximately $67 \%$ (i.e. $15 v .5 \mathrm{~g}$ ) decreased intake by approximately $30 \%{ }^{(8)}$, whilst reducing sip size of regular-energy and noenergy orangeade by $75 \%$ (i.e. $20 v .5 \mathrm{~g}$ ) decreased intake by approximately $29 \%$ and approximately $17 \%$, respectively ${ }^{(6)}$. The result for ad libitum food intake in study one was more modest than these previous studies that have manipulated bite 

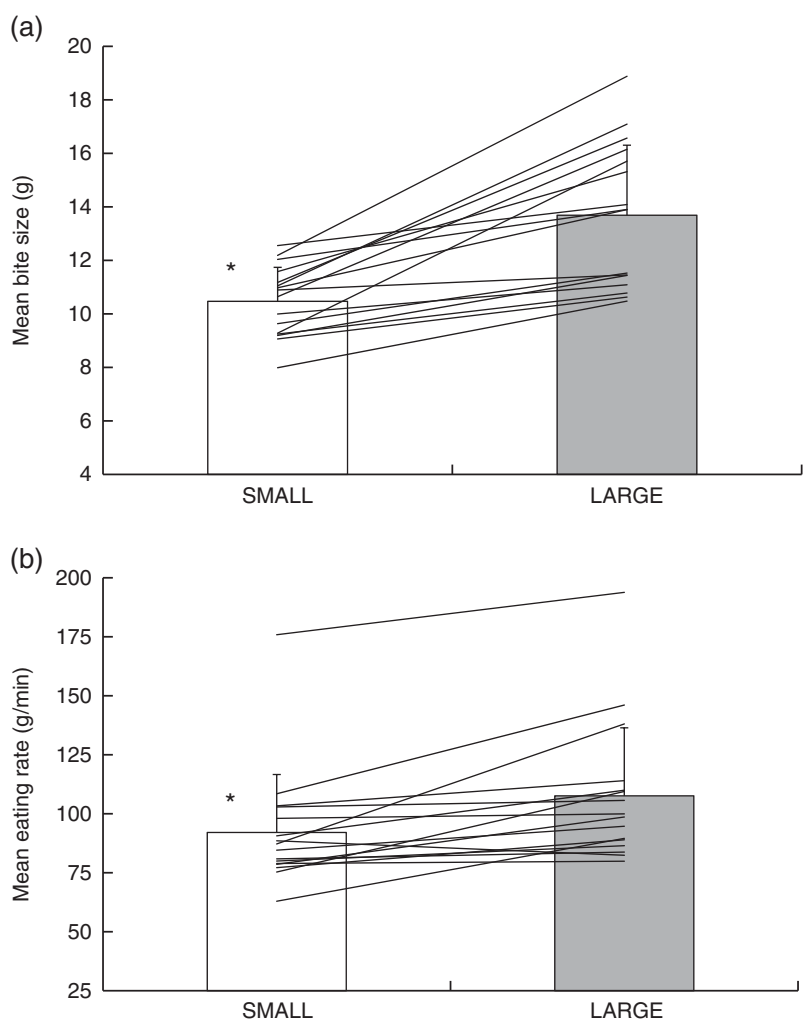

Fig. 2. (a) Mean bite size and (b) mean eating rate during study two. Values are means, with standard deviations represented by vertical bars. Lines are individual subject data. * Significantly different between trials. SMALL, teaspoon; LARGE, dessert spoon.

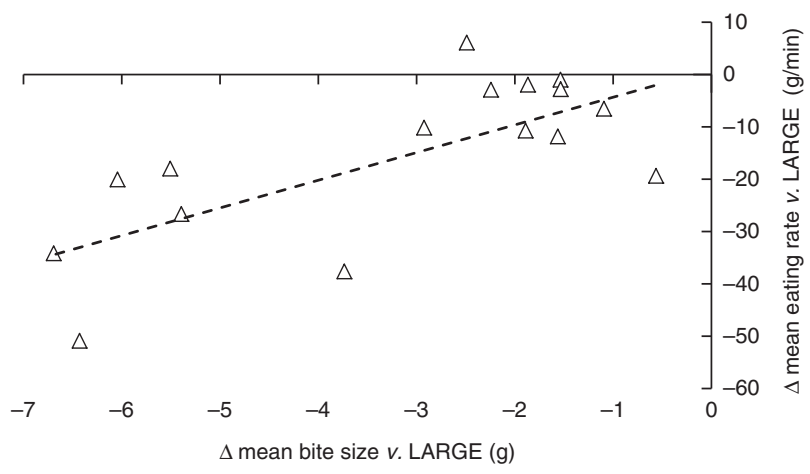

Fig. 3. Change in mean bite size $(\mathrm{g}) v$. change in mean eating rate $(\mathrm{g} / \mathrm{min})$ on the teaspoon (SMALL) trial relative to the dessert spoon (LARGE) trial during study two. Data points are individual subject values. Dashed line represents linear line of best fit.

size (i.e. a reduction of approximately $8 \% v$. approximately $17-30 \%)$, but this is unsurprising given the reduction in bite size observed in study two was also more modest (i.e. a reduction of approximately $24 v \cdot 67-75 \%$ ). These previous studies used a peristaltic pump to deliver the food to the mouth, but study two demonstrates that using a small spoon is a practical method of achieving a meaningful reduction in bite size, and apparently food intake, without the requirement for the individual to consciously reduce their bite size.

Previous studies have reported bite size is associated with eating rate ${ }^{(9,10,20)}$. In study two, subjects took longer to eat the standardised meal when eating with the small spoon, facilitating a reduction of approximately $14 \%$ in mean eating rate. A recent systematic review/meta-analysis reported that eating more slowly was associated with a lower energy intake compared with faster eating ${ }^{(5)}$, and that this was consistent across the various interventions used to alter eating rate. Whilst not all studies that have experimentally manipulated eating rate report reduced energy intake with slower eating ${ }^{(13,21)}$, the majority $\mathrm{do}^{(12,21-25)}$. The change in eating rate between trials was positively associated with the change in bite size, suggesting that the decreased bite size produced by eating with a small spoon may, at least partially, be responsible for the reduced eating rate. Although oral processing time was not measured in the present study, previous work has demonstrated that taking smaller bites leads to a larger number of chews per unit of food $^{(19,26,27)}$. Therefore, the increased number of bites, likely lead to more chewing/oral processing of the food per unit weight, consequently reducing eating rate. Although these elements of eating microstructure were not measured in study one, we propose the cascade of effects observed in study two likely explain the reduction in ad libitum food intake observed in study one.

Interestingly, the manipulation of spoon size appeared to produce diminishing effects as this cascade of eating behaviour responses progressed. The surface area of the small spoon was approximately $39 \%$ less than the large spoon, which caused a decrease in mean bite size of approximately $24 \%$, leading to a reduction in mean eating rate of approximately $14 \%$ and finally a decrease in ad libitum food intake of approximately $8 \%$. As this intervention represented a relatively large reduction in the size of spoon used, the utility of manipulating cutlery size might be limited to relatively small reductions in food intake (i.e. $<10 \%)$. It has been suggested that the discrepancy between energy intake and expenditure causing weight gain is slight ${ }^{(28)}$, and thus even a small difference induced by using smaller cutlery might have a meaningful effect on weight maintenance/ loss goals in the long-term. The studies reported here only tested a relatively small homogenous sample of lean young males. Hopefully these preliminary results will stimulate future research in a much larger and more heterogenous sample including females, children, older adults and those with greater levels of adiposity. Future studies should seek to explore these different groups as well as document responses to repeated exposure to smaller cutlery to explore whether eating behaviour responses are altered by increased exposure, as well as examining the effects of different cutlery types (i.e. fork, knife, etc.).

Manipulation of spoon size did not alter the subjective appetite response to either an ad libitum or a standardised meal. This is consistent with previous studies that have manipulated eating rate, with Robinson et al. ${ }^{(5)}$ reporting that eating more slowly did not affect subjective appetite for ad libitum or standardised meals. The fact that hunger, fullness and DTE were similar at the end of the meal in study one suggests that subjects terminated eating due to satiation, as opposed to boredom or frustration from using the small spoon. Whilst subjects were not specifically asked about their perceptions of using the different size spoons, ratings of satisfaction 
were similar between trials in both study one and study two, possibly suggesting subjects did not find the experience of eating with a small spoon a negative one. However, these satisfaction ratings more likely represent subjects feeling of satisfaction related to their appetite than how satisfied they were with the spoon they ate with. Future work should focus more specifically on how subjects eating experience/enjoyment is affected by manipulation of cutlery size. Nonetheless, given the similarity in sensations of hunger and fullness between trials, it does not appear that subjects in this study terminated eating due to frustration with eating with a small spoon. We attempted to control for demand characteristics in both studies using cover stories and in study one tried to covertly understand who had noticed the difference between conditions through the post-trial interview. Whilst removal of those subjects who reported an awareness of the different spoon sizes did not influence the results for energy intake (Fig. 1(b)), more direct questions about the conditions would have given us a better picture of the success of our cover story and the experience of eating with a small spoon. Interestingly, perhaps future studies should look to blind investigators that interact with subjects too ${ }^{(29)}$, although this might be difficult in the context of the present studies.

The present study used methods that are consistent with the literature exploring eating behaviour responses in a controlled laboratory environment. Whilst this allows relatively small differences between treatments to be detected, it must be acknowledged that the eating situation is not representative of many naturalistic meal environments. Much food intake is planned in advance of eating ${ }^{(30)}$ or is served onto a plate in what the server (whoever that may be) deems to be an appropriate portion. At least for self-served portions, food served is generally eaten in its entirety $^{(31)}$, meaning that in a naturalistic eating setting there may be no opportunity for cutlery size to interact with ad libitum food intake. However, given study two presented here suggests that reducing spoon size reduces bite size and eating rate, the manipulation of cutlery might offer a simple method of manipulating these components of eating behaviour microstructure. It is also worth noting that in some situations where increased energy intake or increased intake of specific foods might be a goal, it may be advantageous to eat with a larger spoon.

In conclusion, the results of these studies demonstrate that eating with a small spoon reduces ad libitum food intake, an effect that is likely caused by alterations in the microstructure of within-meal eating behaviour in lean young men. Specifically, it appears that eating with a small spoon decreases bite size, likely increasing oral processing time, and consequently reducing eating rate. The data reported here suggest using a small spoon might represent a simple practical strategy to reduce bite size, eating rate and ad libitum food intake at a single meal and might be a useful tool that could be used, possibly along with other interventions, to aid in the prevention of weight gain and obesity. Given this study only examined the effect of spoon size on eating behaviour at a single laboratory-based breakfast meal, future studies should examine how different types of cutlery, or different eating occasions/environments influence eating behaviour, as well as how chronic manipulation of cutlery size effects energy intake and energy balance.

\section{Acknowledgements}

This research was partially supported by the National Institute for Health Research Leicester Biomedical Research Centre. This report is independent research by the National Institute for Health Research. The views expressed in this publication are those of the authors and not necessarily those of the NHS, the National Institute for Health Research or the Department of Health.

L. J. J. conceived the study designs, and J. B., T. M., L. J. J. and D. R. B. contributed to the data collection. L. J. J. performed the data analysis, with assistance from D. R. B. L. J. J. wrote the manuscript with assistance from J. B., T. M. and D. R. B.

The authors declare that there are no conflicts of interest.

\section{References}

1. NHS Digital (2017) Statistics on obesity, physical activity and diet, England: 2017. https://www.gov.uk/government/ uploads/system/uploads/attachment_data/file/613532/obesphys-acti-diet-eng-2017-rep.pdf (accessed December 2017).

2. NCD Risk Factor Collaboration (2017) Worldwide trends in body-mass index, underweight, overweight, and obesity from 1975 to 2016: a pooled analysis of 2416 population-based measurement studies in 128.9 million children, adolescents, and adults. Lancet 390, 2627-2642.

3. Hollands GJ, Shemilt I, Marteau TM, et al. (2015) Portion, package or tableware size for changing selection and consumption of food, alcohol and tobacco. Cochrane Database Syst Rev, issue 9, CD011045.

4. Robinson E, Nolan S, Tudur-Smith C, et al. (2014) Will smaller plates lead to smaller waists? A systematic review and meta-analysis of the effect that experimental manipulation of dishware size has on energy consumption. Obes Rev 15, 812-821.

5. Robinson E, Almiron-Roig E, Rutters F, et al. (2014) A systematic review and meta-analysis examining the effect of eating rate on energy intake and hunger. Am J Clin Nutr $\mathbf{1 0 0}$, $123-151$

6. Weijzen PL, Smeets PA \& de Graaf C (2009) Sip size of orangeade: effects on intake and sensory-specific satiation. $\mathrm{Br}$ J Nutr 102, 1091-1097.

7. Zijlstra N, de Wijk RA, Mars M, et al. (2009) Effect of bite size and oral processing time of a semisolid food on satiation. Am J Clin Nutr 90, 269-275.

8. Bolhuis DP, Lakemond CM, de Wijk RA, et al. (2013) Consumption with large sip sizes increases food intake and leads to underestimation of the amount consumed. PLOS ONE $\mathbf{8}$, e53288.

9. de Wijk RA, Zijlstra N, Mars M, et al. (2008) The effects of food viscosity on bite size, bite effort and food intake. Physiol Behav 95, 527-532.

10. Almiron-Roig E, Tsiountsioura M, Lewis HB, et al. (2015) Large portion sizes increase bite size and eating rate in overweight women. Physiol Behav 139, 297-302.

11. Geier AB, Rozin P \& Doros G (2006) Unit bias. A new heuristic that helps explain the effect of portion size on food intake. Psychol Sci 17, 521-525.

12. Andrade AM, Greene GW \& Melanson KJ (2008) Eating slowly led to decreases in energy intake within meals in healthy women. J Am Diet Assoc 108, 1186-1191.

13. Andrade AM, Kresge DL \& Teixeira PJ (2012) Does eating slowly influence appetite and energy intake when water intake is controlled? Int J Behav Nutr Phys Act 9, 135. 
14. Mishra A, Mishra H \& Masters TM (2012) The influence of bite size on quantity of food consumed: a field study. $J$ Consum Res 38, 791-795.

15. Stunkard AJ \& Messick S (1985) The three-factor eating questionnaire to measure dietary restraint, disinhibition and hunger. J Psychosom Res 29, 71-83.

16. Durnin JV \& Womersley J (1974) Body fat assessed from total body density and its estimation from skinfold thickness: measurements on 481 men and women aged from 16 to 72 years. Br J Nutr 32, 77-97.

17. Mifflin MD, St Jeor ST \& Hill LA (1990) A new predictive equation for resting energy expenditure in healthy individuals. Am J Clin Nutr 51, 241-247.

18. Flint A, Raben A, Blundell JE, et al. (2000) Reproducibility, power and validity of visual analogue scales in assessment of appetite sensations in single test meal studies. Int J Obes Relat Metab Disord 24, 38-48.

19. Corney RA, Horina A, Sunderland C, et al. (2015) Effect of hydration status and fluid availability on ad-libitum energy intake of a semi-solid breakfast. Appetite $\mathbf{9 1}$, 399-404.

20. Spiegel TA (2000) Rate of intake, bites, and chews - the interpretation of lean-obese differences. Neurosci Biobehav Rev 24, 229-237.

21. Martin CK, Anton SD, Walden H, et al. (2007) Slower eating rate reduces the food intake of men, but not women: implications for behavioral weight control. Behav Res Ther $\mathbf{4 5}$, 2349-2359.
22. Ioakimidis I, Zandian M, Bergh C, et al. (2009) A method for the control of eating rate: a potential intervention in eating disorders. Behav Res Methods 41, 755-760.

23. Scisco JL, Muth ER, Dong Y, et al. (2011) Slowing bite-rate reduces energy intake: an application of the bite counter device. J Am Diet Assoc 111, 1231-1235.

24. Smit HJ, Kemsley EK, Tapp HS, et al. (2011) Does prolonged chewing reduce food intake? Fletcherism revisited. Appetite 57, 295-298.

25. Karl JP, Young AJ, Rood JC, et al. (2013) Independent and combined effects of eating rate and energy density on energy intake, appetite, and gut hormones. Obesity 21, E244-E252.

26. Nakamichi A, Matsuyama M \& Ichikawa T (2014) Relationship between mouthful volume and number of chews in young Japanese females. Appetite 83, 327-332.

27. Goto T, Nakamich A, Watanabe M, et al. (2015) Influence of food volume per mouthful on chewing and bolus properties. Physiol Behav 141, 58-62.

28. Hall KD, Sacks G, Chandramohan D, et al. (2011) Quantification of the effect of energy imbalance on bodyweight. Lancet 378, 826-837.

29. Gilder TSE \& Heerey EA (2018) The role of experimenter belief in social priming. Psychol Sci 29, 403-417.

30. Brunstrom JM (2011) The control of meal size in human subjects: a role for expected satiety, expected satiation and pre-meal planning. Proc Nutr Soc 70, 155-161.

31. Wansink B \& Cheney MM (2005) Superbowls: serving bowl and food consumption. JAMA 293, 1727-1728. 